\title{
Simulation Analysis on Applicability of Meta Material and PBG Based mm-Wave Planar Antenna for Advanced Cellular Technologies
}

\author{
Smrity Dwivedi \\ The LNM Institute of Information Technology, Rajasthan, India \\ Email: sdwivedi.rs.ece@gmail.com.
}

How to cite this paper: Dwivedi, S. (2017) Simulation Analysis on Applicability of Meta Material and PBG Based mm-Wave Planar Antenna for Advanced Cellular Technologies. Open Journal of Antennas and Propagation, 5, 23-35.

https://doi.org/10.4236/ojapr.2017.51003

Received: January 9, 2017

Accepted: March 5, 2017

Published: March 8, 2017

Copyright $\odot 2017$ by author and Scientific Research Publishing Inc. This work is licensed under the Creative Commons Attribution International License (CC BY 4.0). http://creativecommons.org/licenses/by/4.0/

\begin{abstract}
Metamaterials have got a unique identification in the field of electromagnetic. Left handed metamaterials have been recognized through its working at high frequencies with larger bandwidth in antenna design. The author has proposed a multi substrates LH metamaterials with an array of split triangular antennas for wideband as well as for larger bandwidth. FR4 is being used at frequency $42-26 \mathrm{GHz}$ with thickness of $3 \mathrm{~mm}$ for substrate and matching circuit is inserted to get high gain with minimum reflections. The number of rods in PBG is 100, which is found to get high return losses and ideal behavior of VSWR. Obtained results have been in good agreement such as $S 11$ is approximately $-30 \mathrm{~dB}$, VSWR $1.8 \mathrm{~dB}$ and the bandwidth enhancement is $2 \mathrm{GHz}$ at frequency $5 \mathrm{GHz}$. All results are plotted using commercial software CST microwave studio (Version 2012) and MATLAB.
\end{abstract}

\section{Keywords}

LH Metamaterials, PBG, Planar Antenna, CST Microwave Tools, Return Loss, Radiation Pattern

\section{Introduction}

Growing demand of bandwidth has increased an interest in antenna fields to fit into new era of wireless communication. From large size antennas to very thin and slick antennas, they have been developed according to need and users requirement. Basically, patch antennas played an important role in communication through wireless connections [1]. Not only the variations in parameters of patch antennas have been significant but also characteristics of antenna miniaturization have been developed and planted to improve the radiation properties. One technique, used to reduce the size of antenna is metamaterials based antennas. 
A metamaterial is a kind of artificial synthetic composite material with a specific type of shape and structure, which exhibits properties that are not found in natural materials [2]. Basically, a metamaterial is designed on the basis of periodic structure to get novel electromagnetic properties, e.g. negative permittivity or permeability, zero refractive index, and huge chirality [3] [4]. Like all other common natural materials, the properties of metamaterials are decided by their components and their critical arrangements. In order to achieve specific properties, the components should be designed with specific patterns (periodic), yielding resonant structures. These components, sometimes called meta-atoms or metamolecules, which are periodically arranged in one, two, or three dimensions and all periodic patterns, can be coupled with each other which considerably modify the properties of the metamaterial.

Metamaterials have been widely used in design of microwave devices and antennas. It is flexibility bound to use it for huge number of new devices and antennas. Fabrication and novel performance are being used everywhere and made a vast change to design a smart and advanced antenna for new generation of cellular technology. From radio frequencies to optical frequencies, metamaterials have been designed and realized for different functions, such as negative refractive index, huge chirality, anisotropy and bianisotropy [3] [4]. From the frequency point of view, metamaterials are classified into several categories such as microwave metamaterials, terahetrz metamaterials, and photonic metamaterials. Because of specific arrangement, like 1D, 2D and 3D meramaterials have speciality to enhance all the properties of structures. On the basis of microwave frequencies, there are several important types of metamaterials which are being used in today's era. Veselago media [5] [6], first kind of metamaterials, where real parts of the permittivity and permeability have positive values, is named as DPS (double positive) in upper right corner and DNG (double negative) is mentioned in lower left corner, where both the permittivity and permeability have negative refractive indexes. Second kind of metamaterials is $2 \mathrm{D}$ metamaterials named as high impedance plane, which consists of periodic structure of resonant unit cells [7] [8]. Third type of metamaterials is based on chirality which basically describes an object; especially a molecule that produces a non-superimposible mirror image of itself [9] and due to this, the property changes the polarization of an incident electromagnetic wave. This can be found in $2 \mathrm{D}$ as well as $3 \mathrm{D}$ structures according to usability. Others metamaterials are defined with novel characteristics, (different from others which are mentioned above), e.g. high ani-sotropy [10], large nonlinearity and high dispersion.

Metamaterials having high dispersion and nonlinearity fall into category of electromagnetic band gap arrangement, whose periodicity can be change less than calculated wavelength of given resonant frequency. Such structures are different from LH (left handed) metamaterials and widely known as anisotropic or none resonating or PBG (photonic band gap) structures [11] [12]. Several works have been performed based on PBG structures and are undergoing as to get more and more bandwidth at high frequency. PBG structures are basically the arrange- 
ment of crystals (especially semiconductor) to control the propagation of electromagnetic waves [11] [12]. Electrons behave like a wave for periodic structures according to the quantum mechanics. Similar to LHM, periodic structure that can influence on the electro magentic waves was given different names: photonic crystals (PC), photonoc band gap (PBG), electromagnetic band gap (EBG), microwave band gap (MBG), or simply periodic structure. It is also found in $1 \mathrm{D}$, $2 \mathrm{D}$ and $3 \mathrm{D}$ according to the usage. The most important part of PBG structures are defect [13], which disturbing the periodicity of periodic structures. On account of this, the propagation of electromagnetic waves, pass through the resonant cavity, where defect treated as a cavity. It forms free frequency mode inside the forbidden band-gap during transmission. PBGs can be used for any frequency range, start from radio frequency (RF) to X-rays. Mostly, it is being used in optics and microwaves and they have the most applicable results, but with specific problems according to the nature of the medium and its interaction with electromagnetic waves.

Microwave PBGs have their own specifics that are different from optics. First, the longer wavelength means bigger absolute tolerances than in optics. Secondly, capacitance and inductance are specific properties, not directly seen in optics, because that can vary shape characteristics impedance and make influence on the formation of band gaps. 3-D PBG is complicated for both simulation and realization, due to dependency over the angle of incident and on polarization. $\mathrm{Qu}-$ asi 3-D and 2-D structures are more successful structures and are being used to improve and isolate the antennas characteristics and photonic crystal based waveguide. PBG application improves directivity of antennas. It incorporates mainly: suppression of harmonics, suppression of the surface waves which is the biggest challenge for antenna designers. As surface waves radiate from the roughness of the substrate edges and can make harm to the radiation pattern.

\section{Mathematical Analysis}

As mentioned above in Section I, about the metamaterial and PBG based planar antenna, which has been taken here as microstrip patch antenna with inverted U-shape above the substrate. Microstrip antenna act as resonant cavity with four walls, two are upper and lower short walls (patch and ground) and other two are open ends of antenna (left and right) in general [14]. If the antenna is excited at a resonant frequency, a strong field is set up inside the resonant cavity, and a strong current on the surface of the patch. This produces significant radiation due to fringing fields. Fringing field patterns can be demonstrated by Figure 1 .

In the present paper, author has compared the return loss, VSWR and bandwidth for metamaterial and PBG based antenna and has tried to find out the usability of enhanced bandwidth for $5 \mathrm{G}$ systems.

First to define metamaterial based devices, so many specific models are present, having information about how metamaterials works and importance of their refractive index that is its permittivity and permeability value either both are negative or positive or only one of them is showing negative and positive va- 
lues in coordinate systems as shown in Figure 2, where one can easily dictates the behavior of materials.

One of the famous model to define the properties of metamaterial, is given by Lorentz, which is most usable and well known as Lorentz oscillator model. In absence of electric fields and magnetic fields, electronic charges, ions etc. have not contained any specific direction. In the presence of electric fields or magnetic fields, they become polarized and displaced the electron clouds. Equation (1) is valid for electromagnetic waves used here, to show the motion of electron in the material. In Equation (1), first term in left hand side is inertia, second term is loss and third term is restoring force, whereas, in right hand side denotes the applied electric fields. Due to these terms, electrons get polarized and start working according to electrical force and combined with the EM wave, which is out of phase, and generates oscillation.

$$
m \frac{\partial^{2} r}{\partial t^{2}}+m \Gamma \frac{\partial r}{\partial t}+m \omega_{0}^{2} r=-q E .
$$

The inverted U-Shape planar antenna produces magnetic material like response and exhibits negative permeability, which represent the plasmonic type of frequency in the form of [15] [16].

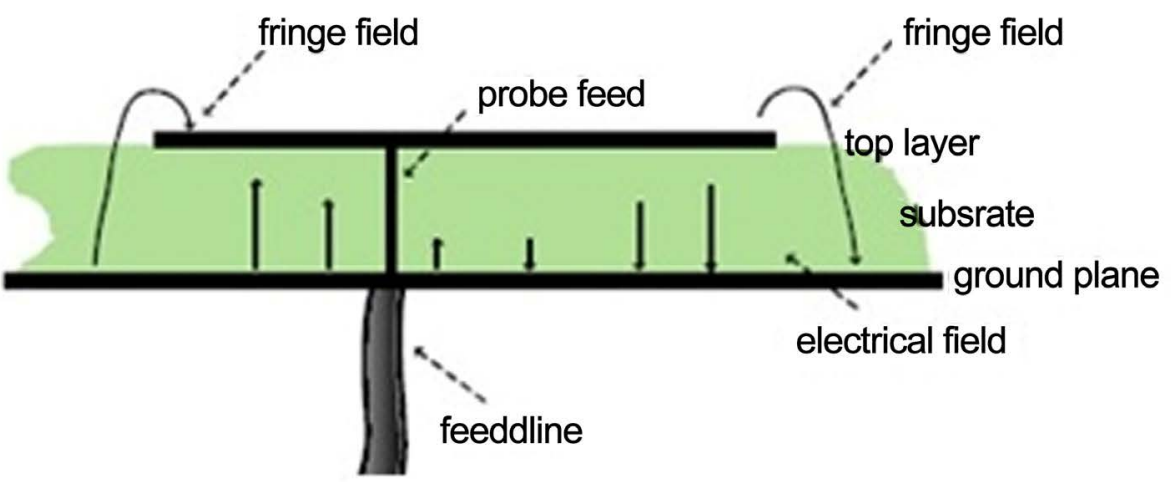

Figure 1. Fringing field pattern in microstrip antenna [14].

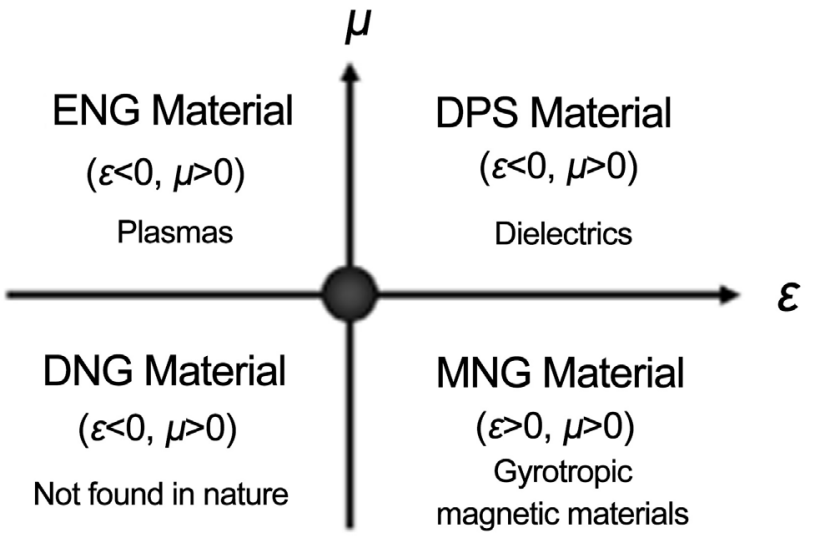

O Zero-index

Figure 2. Classification of metamaterials on the basis of their permittivity and permeability [17]. 


$$
\mu(\omega)=\mu_{0}\left(1-\frac{\omega_{p m}^{2}}{\omega\left(\omega-j \Gamma_{m}\right)}\right)
$$

where, $\omega_{\mathrm{pm}}=$ Magnetic plasma frequency, $\Gamma_{m}=$ Damping coefficient, $\mu_{0}=$ Permeability in free space.

Negative permeability also comes, when, $\omega<\omega_{\mathrm{pm}}$, whereas, capacitive loaded structure is responsible for negative permittivity due to strong dielectric exhibits by this structure [18]. Due to this condition, an electric dipole moment is generated in the structure, which exhibits the plasmonic type of permittivity, function of frequency [19] [20].

$$
\varepsilon(\omega)=\varepsilon_{0}\left(1-\frac{\omega_{p e}^{2}}{\omega\left(\omega-j \Gamma_{e}\right)}\right)
$$

where, $\omega_{\mathrm{pe}}=$ Electric plasma frequency, $\Gamma_{e}=$ Damping coefficient, $\varepsilon_{0}=$ Permittivity in free space.

This structure will also give negative permittivity at, $\omega<\omega_{\mathrm{pe}}$. Equations (2) and (3) are derived using Lorentz oscillator model. But, besides it, one more models, Drude model for metals is being widely used and very easy to apply for measuring out the negative permittivity and permeability [21]. Equation (4) does not have any restoring force part in left hand side, means electron are not bound within the structure, that's why restoring force is equal to zero.

$$
m \frac{\partial^{2} r}{\partial t^{2}}+m \Gamma \frac{\partial r}{\partial t}=-q E
$$

Negative permittivity and permeability can be calculated using Equation (4), which will be function of frequency, widely known as dielectric functions.

$$
\begin{gathered}
\varepsilon_{r}=1-\frac{\omega_{p}^{2}}{\omega^{2}+j \omega \Gamma} . \\
\omega_{p}^{2}=\frac{N q^{2}}{\varepsilon_{0} m} .
\end{gathered}
$$

Equation (5) is being used to calculate the permittivity of material according to Drude model, whereas plasma frequency is related to total number of charges or electron cloud, which is measured from Equation (6).

Like other electromagnetic materials behavior, LHM is also based upon Maxwell's equations $(\nabla \times E=-j \omega(-\mu) H)$ and $(\nabla \times H=j \omega(-\varepsilon) E)$, is properly used here and give a proper direction of magnetic field, electric field as well as polarization. Power and phase are in same direction and polarization is just opposite of fields, which gives negative refractive index of the material. Due to causality, poynting vector corresponds to forward travelling energy, which is just opposite of power and phase, produces a several losses, that can minimize by using special type of structures, inverted U-shape is one of the best and widely used structure under the metamaterials. Due to negative refractive index, structure behavior is changed just opposite of Snell's law. So, waves propagate, having two main components, evanescent and oscillatory, in which evanescent component grows higher as compared to an oscillatory component in LHM. 
For simple rectangular microstrip antenna, the resonance frequency depends on the patch size, cavity dimension, and the filling dielectric constant, given as,

$$
f_{m n}=\frac{k_{m n} c}{2 \pi \sqrt{\varepsilon_{r}}}
$$

where $m, n=0,1,2, \ldots ., k_{\mathrm{mn}}=$ wave number at $m, n$ mode, $c$ is the velocity of light, $\varepsilon_{r}$ is the dielectric constant of substrate, and

$$
k_{m n}=\sqrt{\frac{(m \pi)^{2}}{W}+\frac{(n \pi)^{2}}{L}} .
$$

For $T M_{01}$ mode, the length of non radiating rectangular patch's edge at a certain resonance frequency and dielectric constant according to Equation (7) becomes,

$$
\begin{gathered}
L=\frac{c}{2 f_{r} \sqrt{\varepsilon_{r}}}, \\
W=\frac{c}{f_{r}} \sqrt{\frac{2}{\varepsilon_{r}+1}},
\end{gathered}
$$

where $f_{\mathrm{r}}=$ resonance frequency at which the rectangular microstrip antennas are to be calculated. The radiating edge $W$, patch width, is usually chosen such that it lies within the range $L\langle W\langle 2 L$, for efficient and intense radiation. The ratio $W / L=1.5$ gives good performance according to the side lobe appearances in radiation pattern. In usual practice, the fringing effect causes the effective distance between the radiating edges of the patch to be slightly greater than $L$. Therefore, the actual value of the resonant frequency is slightly less than $f_{\mathrm{r}}$. Taking into account the effect of fringing field, the effective dielectric constant for $T M_{01}$ mode is derived using [22] [23]. Using these equations, total length can be calculated as,

$$
L=\frac{c}{2 f_{r} \sqrt{\varepsilon_{e f f}}}-2 \Delta l
$$

where, $\varepsilon_{\text {eff }}=$ effective dielectric constant and $\Delta I=$ line extension which is given as,

$$
\begin{gathered}
\varepsilon_{\text {eff }}=\frac{\left(\varepsilon_{r}+1\right)}{2}+\frac{\left(\varepsilon_{r}+1\right)}{2}\left[1+12 \frac{h}{W}\right]^{-1 / 2}, \\
\frac{\Delta l}{h}=0.412 \frac{\left(\varepsilon_{\text {eff }}+0.3\right)\left(\frac{W}{h}+0.264\right)}{\left(\varepsilon_{\text {eff }}-0.258\right)\left(\frac{W}{h}+0.8\right)} .
\end{gathered}
$$

Rectangular microstrip is three layered structure, where patch, substrate and ground start working together. A combination of parallel plate radiation conductance and capacitance susceptance loads both radiating edges of the patch, which can be measured as,

$$
G_{1}=\frac{W}{120 \lambda_{0}}\left[1-\frac{\left(k_{0} h\right)^{2}}{24}\right], \frac{h}{\lambda_{0}}<\frac{1}{10},
$$




$$
R_{\text {in }}=\frac{1}{2 G_{1}},
$$

where $\lambda_{0}$ is the free-space wavelength and wave number $k_{0}=\left(2 \pi f_{\mathrm{r}}\right) / c$. The input conductance of the patch fed on the edge will be twice the conductance of one of the edge slots, which can be obtained as.

$$
R_{\text {in }}=\frac{1}{2 G_{1}} \cos ^{2} \frac{\pi}{L} x_{0}, 0 \leq x_{0} \leq L / 2 .
$$

The patch can be fed by any feedings, so the impedance will vary from zero in the center to the edge resistance approximately as.

Now, arranging PBG structure in rectangular patch antenna follows certain rules just because of periodic structure, in which period of PBG structure $T$ is half of the guide wavelength of a general microwave strip $\lambda_{\mathrm{g}}$, can be written as,

$$
T=\lambda_{g} / 2
$$

where,

$$
\begin{gathered}
\lambda_{g}=\frac{c}{f \sqrt{\varepsilon_{e}}}, \\
\varepsilon_{e}=\frac{\left(\varepsilon_{r}+1\right)}{2}+\frac{\left(\varepsilon_{r}-1\right)}{2}\left[1+12 \frac{h}{W}\right]^{-1 / 2} .
\end{gathered}
$$

Here, the rectangular inverted $U$ shape patch antenna is designed in a conventional fashion by itself and then surrounded properly by the PBG lattice structure in substrate. The period of PBG with square lattice is $T=6 \mathrm{~mm}$. Several cases of return loss with different cell size a relative to the period $T$ are simulated, and an optimum size of the hole $a / T=2 / 3$ is measured. The mutual coupling among rectangular conformal microstrip antenna elements and interconnection feeding scheme should be figured out in the design steps. The distance between elements of proposed array is $12.5 \mathrm{~mm}$, integrated with groove loaded microstrip feeding with the depth $l_{\text {slot }}$. For the matching of input impedance with the antenna can be calculated [23],

$$
l_{\text {slot }}=\arccos \left(\sqrt{Z_{c} / R_{a}}\right) \frac{L}{\pi}
$$

where, $Z_{\mathrm{c}}$ is the characteristic impedance of the microstrip, $R_{a}=\left(120 \pi \lambda_{0} h Q\right) /\left(\varepsilon_{r} L W\right), Q$ is the quality factor of all the loss occurred. When simple rectangular microstrip antenna working on $T M_{01}$ mode, $Q$ is obtained as,

$$
Q=\left[\frac{120 \pi h G_{r}}{\varepsilon_{r} L W\left(1-3.4 H_{e}\right)}+\frac{1}{\pi h \sqrt{\frac{\lambda_{0}}{120 \sigma_{e}}}}+\tan \delta\right]^{-1},
$$

while $H_{e}=\sqrt{\varepsilon_{r}-1} h / \lambda_{0}, \quad \sigma_{e}$ is specific conductance; $\tan \delta$ is tangent loss. $G_{\mathrm{r}}$ is radiation conductance of rectangular patch,

$$
G_{r}=\frac{1}{45}\left[\frac{L}{\lambda_{0}}\right]^{2}
$$

in this proposed structure. 


\section{Results and Discussion}

Comparative analysis has been performed between metamaterial and PBG based structure for frequency approximately $44 \mathrm{GHz}$ and results related to this work are properly shown using commercial software CST microwave studio. Parameters have been decided using all equations in Section III, and have been kept same for both design. For metamaterial based antenna design, array of inverted $\mathrm{U}$ has been taken to check the compatibility with $5 \mathrm{G}$ advance cellular system technology. For PBG, rods have been taken, putting at equal periodicity. Parameters are considered according to the resonant frequency given in Table 1.

Design structure of planar antenna using metamaterial and PBG have been shown in Figure 3(a) and Figure 3(b) respectively. From Figure 3(a), it is clear about the shape of radiating patch which is inverted $U$, forming arrays structure to radiate properly and reduces the losses such as surface radiation loss etc. Figure 3 (b) reveals the PBG based structure, where all the parameters are same, only the difference in having rods below the patch, which actually being used to propagate single mode $T M_{01}$ mode including propagation of EM waves through stop band of the structure. This structure especially mends for to remove losses through substrate and create a channel for propagation through a particular stop bands. Substrate material FR-4 shows a better compatibility and removes complexity, compared to other dielectric materials.

Table 1. Design specification of planar antenna.

\begin{tabular}{ccc}
\hline Design Configuration Antenna & Material Used & Parameters \\
\hline Substrate & FR-4 & Height $=3 \mathrm{~mm}$ \\
Patch & Copper (PEC) & Height $=0.4 \mathrm{~mm}$ \\
Ground Thickness & PEC & $L=25 \mathrm{~mm}, W=25 \mathrm{~mm}$, \\
PBG Rods & Vacuum & Height $=0.4 \mathrm{~mm}$ \\
Microstrip Transmission Line & PEC & 100 Periodicity $=4 \mathrm{~mm}$ \\
& & Calculated Using Equations and \\
\end{tabular}

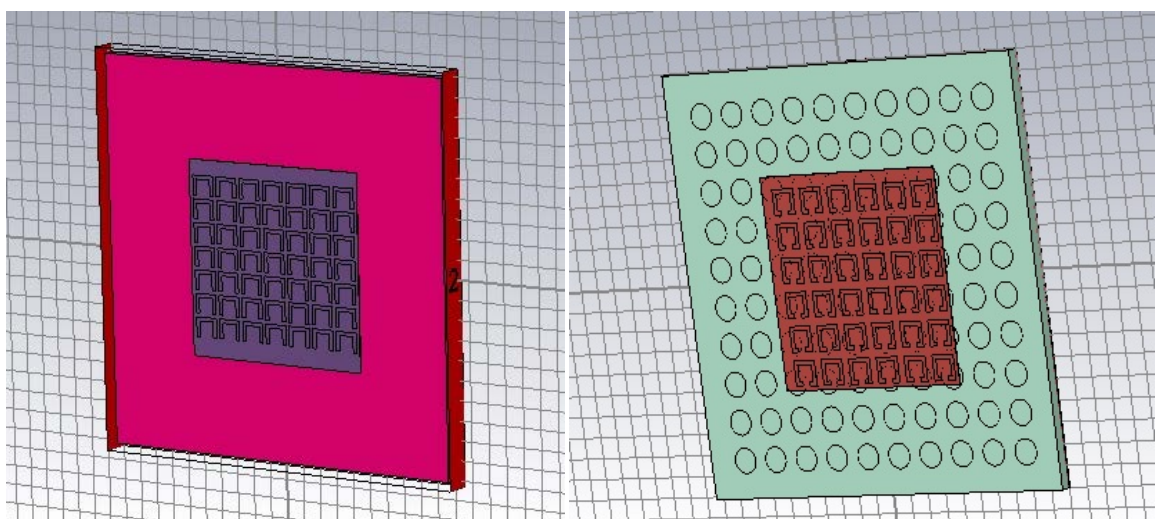

(a)

(b)

Figure 3. Left handed metameterial based planar antenna (a); PBG based planar antenna (b). 
The return loss for both types of structures is most important parameter to reveal its applicability in mobile field communication. As shown in Figure 4(a) and Figure 4(b), return loss is more improved in case of metamaterial antenna, as compared with PBG antenna. From Figure 4(a), return loss is minimum at $44.18 \mathrm{GHz}$, has value $-30 \mathrm{~dB}$, whereas in Figure $4(\mathrm{~b})$, return loss is minimum at $43.28 \mathrm{GHz}$ as well as at $44.5 \mathrm{GHz}$, but not comparable with Figure $4(\mathrm{a})$. Values at these two frequencies are $-23 \mathrm{~dB}$ and $-24 \mathrm{~dB}$ respectively. Although, Figure 4 (b) is giving multiband property, but because of losses, noise signal (reflection noise) is responsible for such return loss.

S-Parameter [Magnitude in dB]

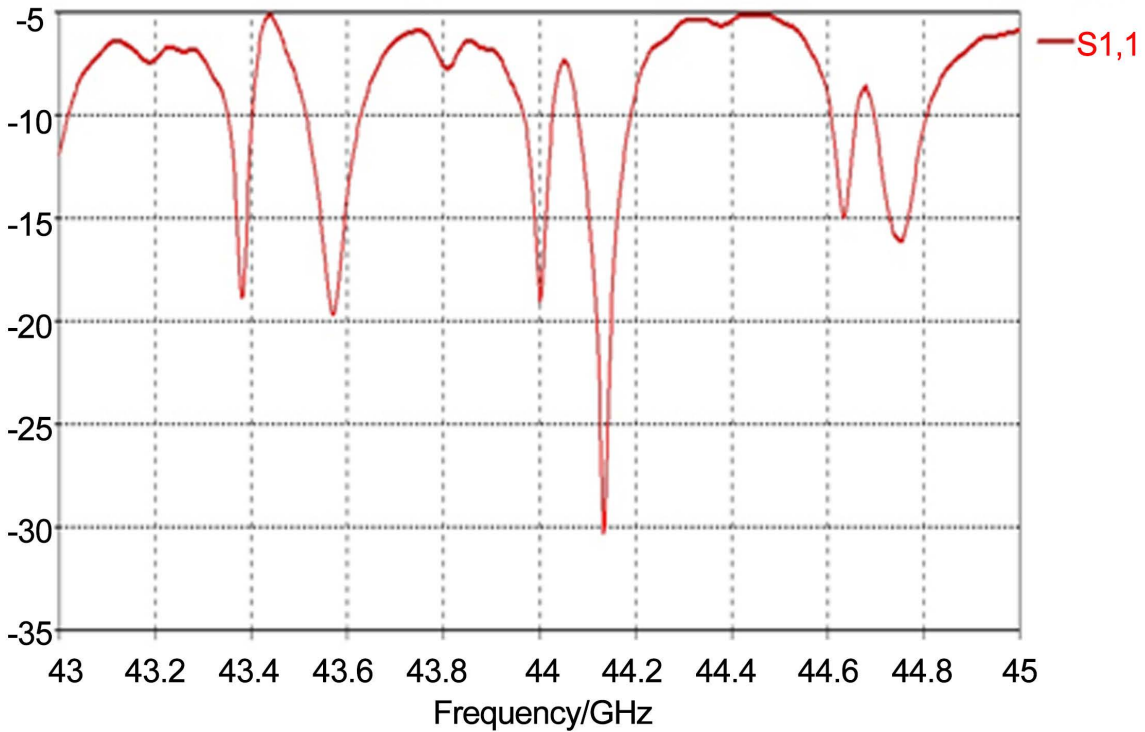

(a)

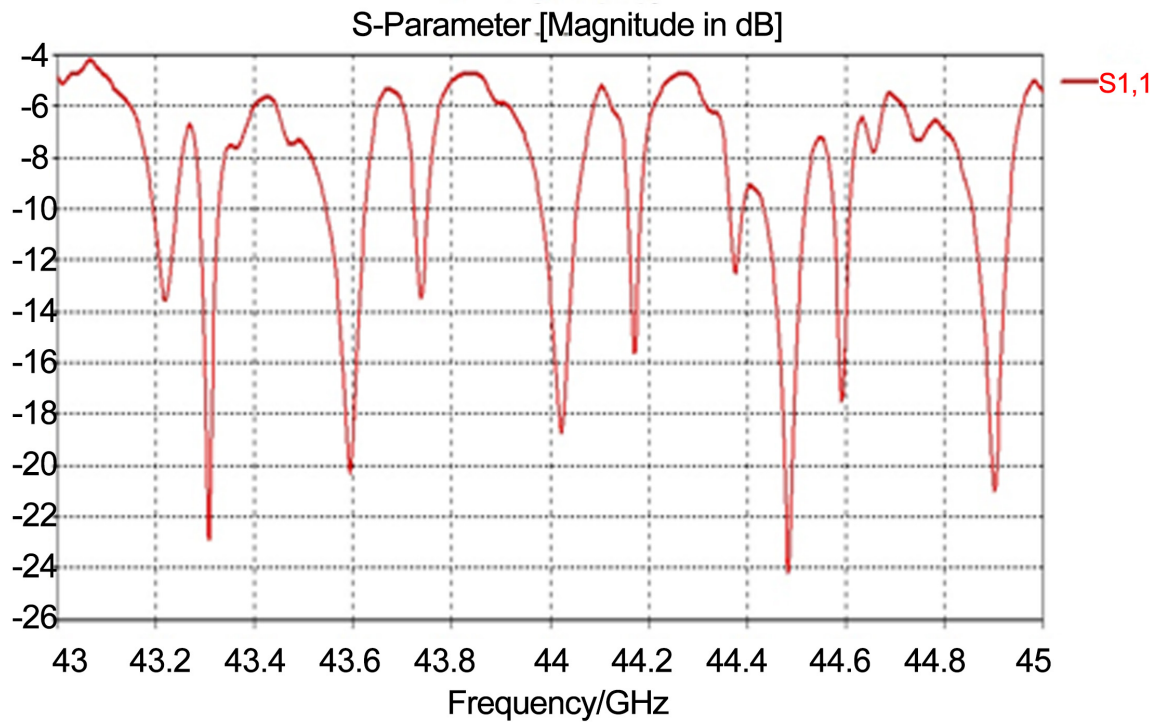

(b)

Figure 4. Return loss for metamaterial antenna (a), return loss for PBG antenna (b). 
One of the important parameter is VSWR, which proposed antenna compatibility with transmission from input to output and gives information about reflection from output in form of standing wave ratio. Ideal value of VSWR is one, which can be achieved only on zero reflection, which is not possible in case planar antennas. Figure 5(a) and Figure 5(b) show the value of VSWR at the resonant frequencies. It is very clear from plots, the VSWR is better in case of metamaterial antenna. In the plot, passes have been taken during simulation to get more accurate results under CST microwave studio.

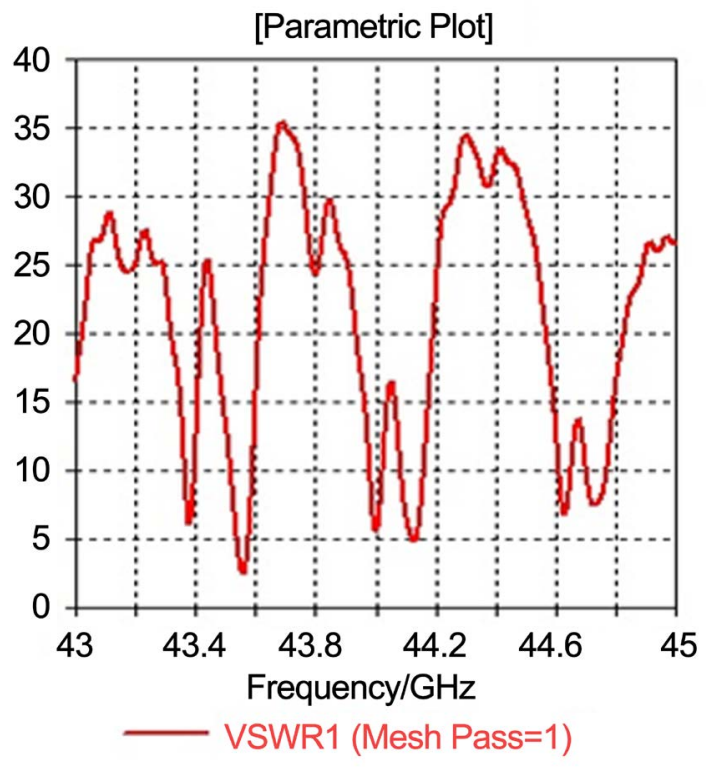

(a)

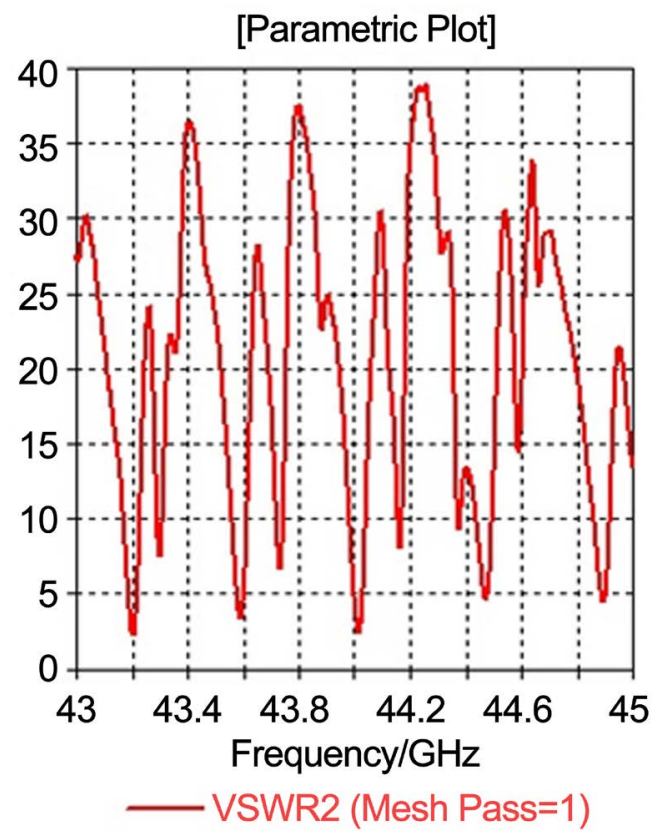

(b)

Figure 5. VSWR of metamaterial inverted U shape antenna (a); VSWR of PBG antenna (b). 
Total efficiency and radiation intensity of planar antennas give directivity, gain, field pattern (Electric and Magnetic) and spectral power density. From Figures 6(a)-6(d), efficiency of both antennas directly measured using simulated plots. From the data, given below in the Plots 6(a) and (b) much more informative in terms of gain, where metamaterial planar antenna is giving higher gain as compared to PBG antenna.

Comparative analysis between metamaterial and PBG antenna, designed at same high frequency to use it for $5 \mathrm{G}$ advanced technology, is given in Table 2.

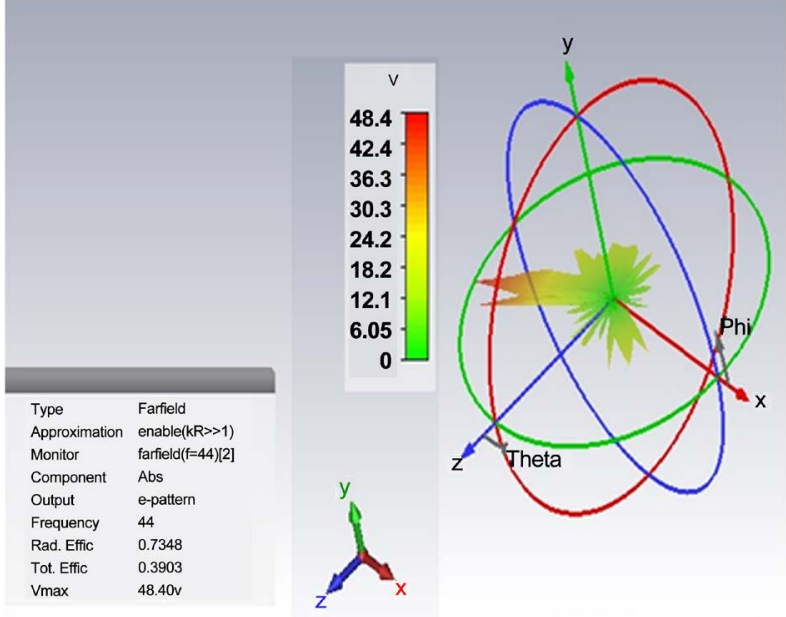

(a)

Farfield Dirctivity Ludwing 3 Right Phase (Phi=90)

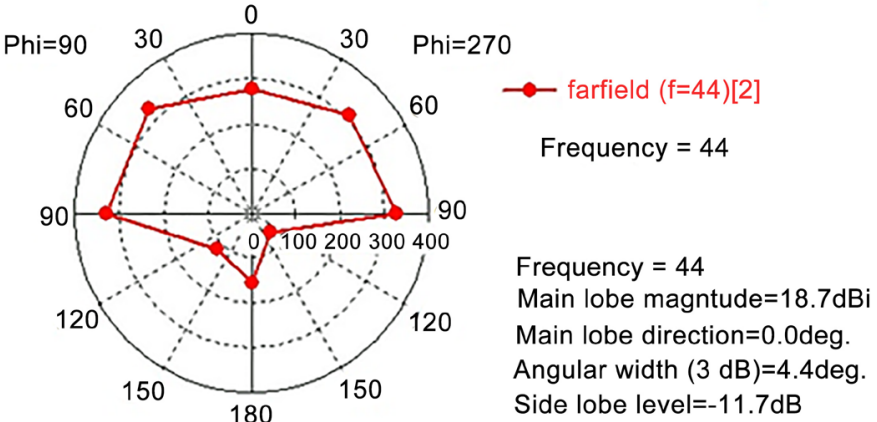

Theta/Degree vs. deg.

(c)

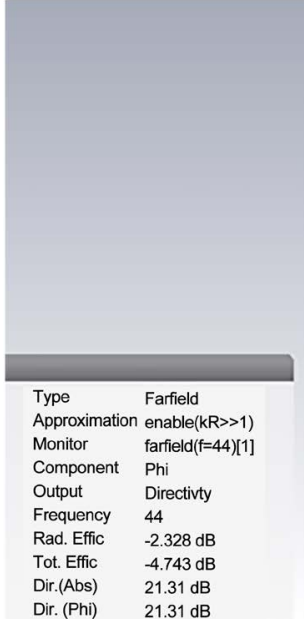

$131 \mathrm{~dB}$

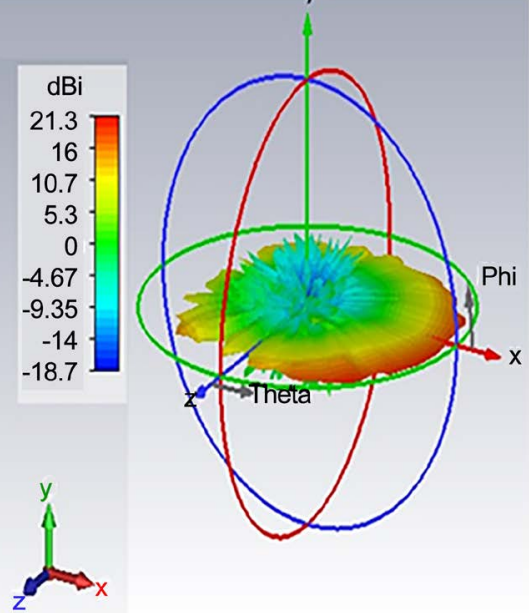

(b)

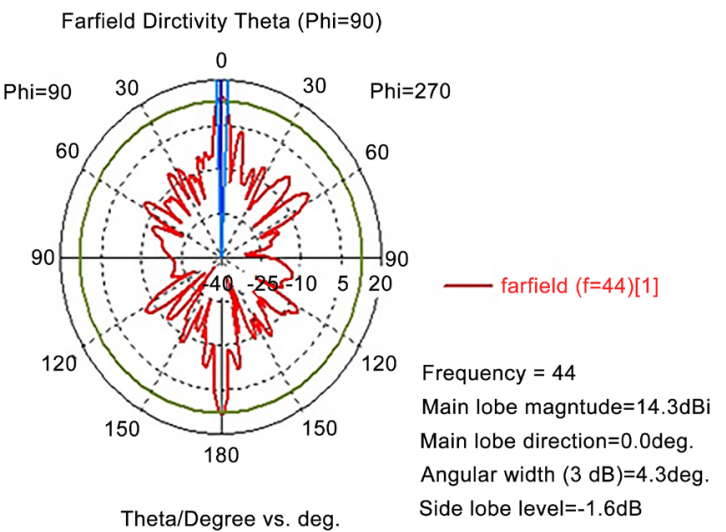

(d)

Figure 6. Total and radiation efficiency of metamaterial antenna (a); Total and radiation intensity of PBG antenna (b); Farfield pattern for metamaterial antenna (c); Farfield pattern of PBG antenna (d).

Table 2. Comparison chart between metamaterial and PBG planar antenna.

\begin{tabular}{ccc}
\hline Design Configuration Antenna & Metamaterial & PBG \\
\hline Return Loss & $-30 \mathrm{~dB}$ & $-26 \mathrm{~dB}$ \\
Bandwidth & $0.2 \mathrm{GHz}$ & $0.15 \mathrm{GHz}$ \\
Gain & $79 \%$ & $60 \%$ \\
VSWR & $1.8 \mathrm{~dB}$ & $1.5 \mathrm{~dB}$ \\
\hline
\end{tabular}




\section{Conclusion}

From entire analysis, it has been understood that metamaterial based antennas is more useful for $5 \mathrm{G}$ advanced communication systems, with proper results and its discussion. The metamaterial inverted $U$ shape antenna has more impact in terms of return loss, VSWR, gain and bandwidth compared with PBG planar antennas. And it is also easy to fabricate for experimental analysis. Efficiency is better in case of metamaterial based antenna as calculated 79\%. But, all results obtained here, are simulated one using CST Microwave studio (Version 2012), but not measured from VNA. This has to be done in future.

\section{Acknowledgements}

The author (Smrity Dwivedi) is thankful for continuous support of her supervisor Prof. P. K. Jain, who has indirectly motivated her.

\section{References}

[1] Ziolkowski, R.W. and Erentok, A. (2006) Metamaterial-Based Efficient Electrically Small Antennas. IEEE Transactions on Antennas and Propagation, 54, 2113-2130. https://doi.org/10.1109/TAP.2006.877179

[2] Wu, B.-I., Wang, W., Pacheco, J., Chen, X., Grzegorczyk, T. and Kong, J.A. (2005) A Study of Using Metamaterials as Antenna Substrate to Enhance Gain. Progress in Electromagnetics Research, 51, 295-328. https://doi.org/10.2528/PIER04070701

[3] Sihvola, A. (2007) Metamaterials in Electromagnetics. Metamaterials, 1, 2-11. https://doi.org/10.1016/j.metmat.2007.02.003

[4] Lapine, M. and Tretyakov, S. (2007) Contemporary Notes on Metamaterials. IET Microwaves, Antennas \& Propagation, 1, 3-11. https://doi.org/10.1049/iet-map:20050307

[5] Mandelstam, J.B. (1945) Group Velocity in Crystal Lattice. Zhurn. Eksp. Teor. Fiz, $15,475-478$.

[6] Veselago, V.G. (1968) The Electrodynamics of Substances with Simultaneously Negative Values of $\varepsilon$ and $\mu$. Soviet Physics Uspekhi, 10, 509-514. https://doi.org/10.1070/PU1968v010n04ABEH003699

[7] Sievenpiper, D., Zhang, L., Broas, R.F., et al. (1999) High-Impedance Electromagnetic Surfaces with a Forbidden Frequency Band. IEEE Transactions on Microwave Theory and Techniques, 47, 2059-2074. https://doi.org/10.1109/22.798001

[8] Vallecchi, A., De Luis, J.R., Capolino, F., et al. (2012) Low Profile Fully Planar Folded Dipole Antenna on a High Impedance Surface. IEEE Transactions on Antennas and Propagation, 60, 51-62. https://doi.org/10.1109/TAP.2011.2167912

[9] Ye, Y. and He, S. (2010) $90^{\circ}$ Polarization Rotator Using a Bilayered Chiral Metamaterial with Giant Optical Activity. Applied Physics Letters, 96, 2001-2035. https://doi.org/10.1063/1.3429683

[10] Serdyukov, A., Semchenko, I., Tretyakov, S., et al. (2001) Electromagnetics of Bianisotropic Materials: Theory and Applications. Gordon and Breach, Amsterdam.

[11] Gorkunov, M. and Lapine, M. (2004) Tuning of a Nonlinear Metamaterial Band Gap by an External Magnetic Field. Physical Review B, 70, Article ID: 235109. https://doi.org/10.1103/PhysRevB.70.235109

[12] Shadrivov, I.V., Kozyrev, A.B., Van der Weide, D.W., et al. (2008) Tunable Transmission and Harmonic Generation in Nonlinear Metamaterials. Applied Physics 
Letters, 93, Article ID: 161903. https://doi.org/10.1063/1.2999634

[13] Yunus, M., Zulkifli, F.Y. and Rahardjo, E.T. (2016) Radiation Characteristics of a Novel $\mu$ Negative Metamaterial Spiral Resonator Antenna at the $2.4 \mathrm{GHz}$. Open Journal of Antennas and Propagation, 4, 1-11. https://doi.org/10.4236/ojapr.2016.41001

[14] Krauss, J.D. (2001) Antennas for All Applications. 3rd Edition, McGraw-Hill, New York.

[15] Dong, Y.D. (2012) Metamaterial-Based Antennas. Proceedings of the IEEE, 100, 2271-2285.

[16] Hwang, R.B., Liu, H.W. and Chin, C.Y. (2009) A Metamaterial-Based E-Plane Horn Antenna. Progress in Electromagnetics Research, 93, 275-289. https://doi.org/10.2528/PIER09050606

[17] Li, L.-W., Li, Y.-N., Yeo, T.S., Mosig, J.R. and Martin, O.J.F. (2010) A Broadband and High-Gain Metamaterial Microstrip Antenna. International Conference on Chinese Physics Letter.

[18] Majid, H.A., Rahim, M.K.A. and Masri, T. (2009) Microstrip Antenna's Gain Enhancement Using Left Handed Metamaterial Structure. Progress in Electromagnetics Research M, 8, 235-247. https://doi.org/10.2528/PIERM09071301

[19] Deshmukh, A.A. and Ray, K.P. (2012) Formulation of Resonance Frequencies for Dual-Band Slotted Rectangular Microstrip Antennas. IEEE Antennas and Propagation Magazine, 54, 78-97. https://doi.org/10.1109/MAP.2012.6309159

[20] Alu, A., Engheta, N., Erentok, A., et al. (2007) Single-Negative, Double-Negative, and Lowindex Metamaterials and Their Electromagnetic Applications. IEEE Antennas and Propagation Magazine, 49, 23-36. https://doi.org/10.1109/MAP.2007.370979

[21] Padilla, W.J., Basov, D.N. and Smith, D.R. (2006) Negative Refractive Index Metamaterials. Materials Today, 9, 28-35. https://doi.org/10.1016/S1369-7021(06)71573-5

[22] Tarot, A.-C., Collardey, S. and Mahdjoubi, K. (2003) Numerical Studies of Metallic PBG Structures. Progress in Electromagnetics Research, 41, 133-157. https://doi.org/10.2528/PIER02010806

[23] Wang, X., Zhang, M. and Wang, S.-J. (2011) Practicability Analysis and Application of PBG Structures on Cylindrical Conformal Microstrip Antenna and Array. Progress in Electromagnetics Research, 115, 495-507. https://doi.org/10.2528/PIER11031703 
Submit or recommend next manuscript to SCIRP and we will provide best service for you:

Accepting pre-submission inquiries through Email, Facebook, LinkedIn, Twitter, etc. A wide selection of journals (inclusive of 9 subjects, more than 200 journals)

Providing 24-hour high-quality service

User-friendly online submission system

Fair and swift peer-review system

Efficient typesetting and proofreading procedure

Display of the result of downloads and visits, as well as the number of cited articles Maximum dissemination of your research work

Submit your manuscript at: http://papersubmission.scirp.org/

Or contact ojapr@scirp.org 